\title{
Effect of a Non-Calcium-Based Phosphate Binder on Fibroblast Growth Factor 23 in Chronic Kidney Disease
}

\author{
Christin Spatz $^{\mathrm{a}}$ Kevin Roe $^{\mathrm{a}}$ Erik Lehman $^{\mathrm{b}}$ Navin Verma ${ }^{\mathrm{a}}$ \\ aPenn State Milton S. Hershey Medical Center, and ${ }^{b}$ Penn State College of Medicine, Hershey, Pa., USA
}

\section{Key Words}

Chronic kidney disease - Fibroblast growth factor 23 .

Hyperphosphatemia - Non-calcium-based phosphate binder - Sevelamer carbonate

\begin{abstract}
Background: Elevated fibroblast growth factor 23 (FGF23) levels are associated with progression of chronic kidney disease (CKD) and increased mortality. Studies in individuals without CKD suggest that FGF23 levels are regulated by dietary phosphorus; however, the effect of pharmacologic phosphorus restriction on FGF23 in CKD patients is uncertain. Methods: We performed a prospective cohort study examining the effect of phosphorus reduction with sevelamer carbonate on FGF23 levels in CKD patients. Adults with an estimated glomerular filtration rate $<60 \mathrm{ml} / \mathrm{min} / 1.73 \mathrm{~m}^{2}$ according to MDRD (Modification of Diet in Renal Disease) and hyperphosphatemia were enrolled. Subjects were started on sevelamer carbonate $800 \mathrm{mg}$ by mouth with meals and the dose was titrated to achieve a serum phosphorus between 2.7 and $4.6 \mathrm{mg} / \mathrm{dl}$ for those with CKD stages III and IV, and between 3.5 and $5.5 \mathrm{mg} / \mathrm{dl}$ for CKD stage V. FGF23 levels were measured at baseline and 3 months. Results were analyzed as percent change from baseline. Results: 40 patients completed the study. Mean estimated glomerular filtration rate by MDRD at entry was $21.2 \pm 10.5$, serum phosphorus
\end{abstract}

$4.8 \pm 0.8$, and FGF23 level $602.3 \pm 1,074.6$. Mean serum phosphorus and FGF23 levels after 3 months were $4.4 \pm 0.9$ and $599.2 \pm 720.9$, respectively. No significant difference was seen in FGF23 $(p=0.76)$ despite a significant difference in phosphorus $(p=0.001)$. Conclusion: The patients treated with sevelamer carbonate did not have a significant change in plasma FGF23 levels despite a significant reduction in phosphorus. It is possible that once overt hyperphosphatemia develops, FGF23 levels may not be reduced by phosphorus reduction alone in CKD patients.

Copyright $\odot 2013$ S. Karger AG, Base

\section{Introduction}

Abnormal mineral metabolism is a key finding nearly universal to those with advanced chronic kidney disease (CKD). Elevated phosphorus levels contribute to arterial calcification, myocardial hypertrophy and endothelial dysfunction $[1,2]$. Studies have shown an independent association between elevated phosphorus levels and mortality in those with CKD as well as the general population $[3,4]$.

This study was presented, in part, at the 2012 National Kidney Foundation Spring Clinical Meetings.

\section{KARGER}

E-Mail karger@karger.com

www.karger.com/nec
(C) 2013 S. Karger AG, Basel

$1660-2110 / 13 / 1232-0061 \$ 38.00 / 0$
Christin Spatz, MD

Penn State Milton S. Hershey Medical Center

500 University Drive, H040, Room C5830

Hershey, PA 17033 (USA)

E-Mail cspatz@hmc.psu.edu 
Recent studies suggest that fibroblast growth factor 23 (FGF23), a phosphaturic hormone, may play an early part in the pathogenesis of abnormal mineral metabolism [5]. FGF23 is produced by osteocytes and directly affects the kidney by downregulating the production of 1,25-vitamin $\mathrm{D}_{3}$ and the expression of $2 \mathrm{a}$ and $2 \mathrm{c}$ sodium-phosphate co-transporters in response to phosphate overload [6]. Elevated FGF23 levels are well documented in patients with CKD and are inversely related to glomerular filtration rate. The increase in FGF23 in CKD patients is felt to be a compensatory response to maintain phosphorus within normal limits when dietary intake exceeds the kidney's ability to excrete phosphorus [5]. Recent studies have demonstrated that increases in plasma FGF23 levels occur in early stages of CKD before phosphorus and parathyroid hormone (PTH) become abnormal [7, 8]. FGF23 has been proposed to be a better marker of the metabolic derangements of CKD since there is minimal circadian and postprandial variation compared to phosphorus and PTH [9-11].

Numerous studies have shown that FGF23 is independently associated with mortality in kidney transplant recipients, new hemodialysis patients and those with CKD [12-14]. In addition, a correlation has been seen between elevated FGF23 levels and progression of CKD [15]. These studies suggest that FGF23 may serve as a biomarker to predict those at highest risk of progression and worse outcomes. More importantly, FGF23 may be a novel target to impact the early mineral derangements that lead to adverse outcomes.

To date, there is a paucity of data examining the effects of phosphorus lowering by pharmacologic means on plasma FGF23 levels. One study performed with dialysis patients did show a decrease in FGF23 with the use of sevelamer [16]. Data on CKD patients is limited by shortduration, small-size studies primarily conducted in normophosphatemic patients [17-19]. Our study focused on hyperphosphatemic patients and was designed to test the hypothesis that prolonged phosphorus reduction by use of a non-calcium-based phosphate binder can significantly lower FGF23 levels in CKD pre-dialysis patients with hyperphosphatemia.

\section{Subjects and Methods}

\section{Study Design}

This study included subjects with stable renal function, ages 18 and older with pre-dialysis CKD stages 3-5 determined by the MDRD (Modification of Diet in Renal Disease) equation. Patients with CKD III and IV were eligible if the serum phosphorus was $\geq 4.6 \mathrm{mg} / \mathrm{dl}$ and with CKD stage $\mathrm{V}$ if phosphorus was $\geq 5.6 \mathrm{mg} / \mathrm{dl}$. Subjects were recruited from the CKD Clinic at Penn State Milton S. Hershey Medical Center between July 2010 and April 2012. Those with a history of renal transplant, major gastrointestinal surgery, currently pregnant, suffered from dysphagia, swallowing disorders, gastrointestinal motility disorders or severe constipation were excluded. Informed consent was obtained from all patients. This study was approved by the local institutional review board and registered with clinical trials.gov NCT01308242.

This was a prospective study conducted over a 3-month period, which involved titration of sevelamer carbonate (Renvela ${ }^{\circledR}, 800$ mg tablets; Genzyme Co., Cambridge, Mass., USA). After obtaining consent, patients received instructions on a low phosphorus diet verbally from study investigators and through educational materials. Those subjects previously on a phosphate binder underwent a 6-week washout. All subjects had calcium, phosphorus, creatinine, plasma FGF23, intact PTH (iPTH) and 25-OH vitamin D levels measured at baseline. Each patient received sevelamer carbonate at a starting dose of $800 \mathrm{mg}$ three times daily with meals. Monthly calcium, phosphorus and creatinine were measured. The dose of sevelamer carbonate was titrated to achieve a serum phosphorus level between 2.7 and $4.6 \mathrm{mg} / \mathrm{dl}$ for those with CKD stages III and IV and between 3.5 and $5.5 \mathrm{mg} / \mathrm{dl}$ for CKD stage V. After 3 months, calcium, phosphorus, creatinine, plasma FGF23, iPTH and $25-\mathrm{OH}$ vitamin $\mathrm{D}$ levels were measured again. During the trial, active vitamin $\mathrm{D}$ doses were held constant and no intravenous iron infusions were administered.

\section{Laboratory Evaluation}

Blood samples for iPTH and plasma FGF23 were taken after an overnight fast of $8 \mathrm{~h}$. All samples were processed by a central laboratory at the Penn State Milton S. Hershey Medical Center except plasma FGF23 levels, which were processed by Mayo Clinic. Routine chemistries were measured in the blood using standard assays. PTH was measured using a bio-iPTH assay that detects the intact 1-84 PTH peptide (Immunotopics, Inc., San Clemente, Calif., USA). At that time of initiation of our study, studies suggested a high correlation among intact and C-terminal assays of FGF23 in CKD patients; thus, measurement of FGF23 via the C-terminal assay (Immunotopics, Inc.) was used in this study. A normal FGF23 level is $<50 \mathrm{RU} / \mathrm{ml}$.

\section{Statistical Analysis}

This study did not use an intent-to-treat analysis. Demographic characteristics and biochemical parameters were described as mean \pm SD. Change from baseline was compared using the paired $t$ test for all variables except FGF23 and 25-OH vitamin D levels, which were analyzed using the Wilcoxon signed-rank test because of skewed distributions. Statistical analyses were performed using SAS. $\mathrm{p}<0.05$ was considered significant.

\section{Results}

A total of 47 patients were enrolled in this study. Two of the patients withdrew during the study due to nonadherence with the study protocol and 4 withdrew due to gastrointestinal symptoms and were excluded from anal-
Spatz/Roe/Lehman/Verma 
ysis. One patient was declared an outlier based on extreme FGF23 levels and was excluded. A total of 40 subjects completed the study and were analyzed. Table 1 summarizes the baseline characteristics of the study population. The mean age was $70 \pm 14$ with a slight female predominance. Approximately one quarter of the patients were on a binder and three quarters on active vitamin $\mathrm{D}$. The main cause of CKD determined by review of medical history was diabetes mellitus (39\%), followed by hypertension (27\%), cardiorenal (12\%) and vascular disease $(7 \%)$. The baseline estimated glomerular filtration rate as measured by the MDRD was $21.2 \pm 10.5 \mathrm{ml} /$ $\min / 1.73 \mathrm{~m}^{2}$.

The mean values for the biochemical parameters observed at baseline and 3 months are shown in table 2. After treatment with sevelamer carbonate, there was a significant reduction in serum phosphorus at the end of 3 months from 4.8 to $4.4 \mathrm{mg} / \mathrm{dl}(\mathrm{p}=0.001) .15$ patients (38\%) required dose titration to $1,600 \mathrm{mg}$ with meals during the study. There was also a reduction in the mean serum iPTH from 162 to $142 \mathrm{pg} / \mathrm{ml}$, however the result was not statistically significant. Those treated with sevelamer, however, did have a significant decrease in $25-\mathrm{OH}$ vitamin D levels from 26.1 to 22.3 ( $\mathrm{p}=0.004$ ).

Figure 1 shows the percentage changes in serum phosphorus, and figure 2 the percentage changes in plasma FGF23 levels in sevelamer-treated patients. There was a small but non-significant reduction in mean plasma FGF23 levels $(602-599 \mathrm{RU} / \mathrm{ml}, \mathrm{p}=0.9)$ in these patients despite significant reduction in serum phosphorus levels.

\section{Discussion}

This study has shown that FGF23 levels did not significantly decrease in patients with advanced CKD despite significant phosphorus reduction. The other studies examining the effects of dietary and pharmacologic phosphorus reduction on plasma FGF23 levels in CKD patients had conflicting results. Oliveira et al. [17] have shown a significant reduction in FGF23 in normophosphatemic patients treated with sevelamer hydrochloride after a 6-week period that was not seen in patients treated with calcium acetate. This trial was limited by small size and many exclusion criteria.

A pilot study evaluating dietary phosphorus restriction and use of lanthanum carbonate on FGF23 in normophosphatemic CKD III and IV patients showed no significant reduction in FGF23 despite a significant reduction in urinary phosphorus [20]. A follow-up study was
Table 1. Baseline demographical, clinical and biochemical parameters of the study population $(n=40)$

\begin{tabular}{lc}
\hline Age, years & $70 \pm 14$ \\
Male gender & $44 \%$ \\
Estimated GFR, ml/min/1.73 m² & $21.2 \pm 10.5$ \\
Cause of CKD & \\
$\quad$ Diabetes mellitus & $39 \%$ \\
$\quad$ Hypertension & $27 \%$ \\
$\quad$ Cardiorenal & $12 \%$ \\
$\quad$ Vascular & $7 \%$ \\
$\quad$ Other (GN, PCKD, ARF, obstruction) & $15 \%$ \\
Phosphate binder use at study entry (yes vs. no) & $24 \%$ \\
Active vitamin D use at study entry (yes vs. no) & $76 \%$ \\
Calcium, mg/dl & $9.3 \pm 0.7$ \\
Phosphorus, mg/dl & $4.8 \pm 0.8$ \\
PTH, pg/ml & $162 \pm 127$ \\
25-OH vitamin D, ng/ml & $26 \pm 1$ \\
\hline
\end{tabular}

Table 2. Biochemical parameters at baseline and 3 months after treatment with sevelamer carbonate $(n=40)$

\begin{tabular}{lccl}
\hline & Baseline & Month 3 & p value \\
\hline Phosphorus, mg/dl & $4.84 \pm 0.8$ & $4.44 \pm 0.9$ & 0.001 \\
FGF23, RU/ml & $602.3 \pm 1,074$ & $599.2 \pm 720.9$ & 0.9 \\
25-OH vitamin D, ng/ml & $26.1 \pm 10.6$ & $22.3 \pm 8.4$ & 0.004 \\
PTH, pg/ml & $162 \pm 127$ & $142 \pm 114$ & 0.26 \\
$\begin{array}{l}\text { Estimated GFR } \\
\quad \mathrm{ml} / \mathrm{min} / 1.73 \mathrm{~m}^{2}\end{array}$ & $21.2 \pm 10.5$ & $21.4 \pm 11.0$ & 0.77 \\
\hline
\end{tabular}

conducted with a $2 \times 2$ factorial, placebo-controlled study of normophosphatemic CKD III and IV patients that showed only the combination of lanthanum carbonate and dietary phosphorus restriction could lower FGF23 levels [19].

In contrast to the prior studies in normophosphatemic subjects, this study attempted to answer whether sevelamer carbonate could lower FGF23 levels in the CKD stage 3-5 patients with hyperphosphatemia. Although serum phosphorus reduction was achieved, no significant reduction in plasma FGF23 levels was seen. Several possible explanations may account for these findings. Since overt hyperphosphatemia is a late manifestation of abnormal mineral metabolism, it is possible that chronically elevated FGF23 levels may not be suppressed by phosphorus reduction alone. Another plausible explanation is that greater and more sustained phosphorus reduction is necessary to achieve a significant decrease in FGF23 levels. The assay type may have also impacted our 


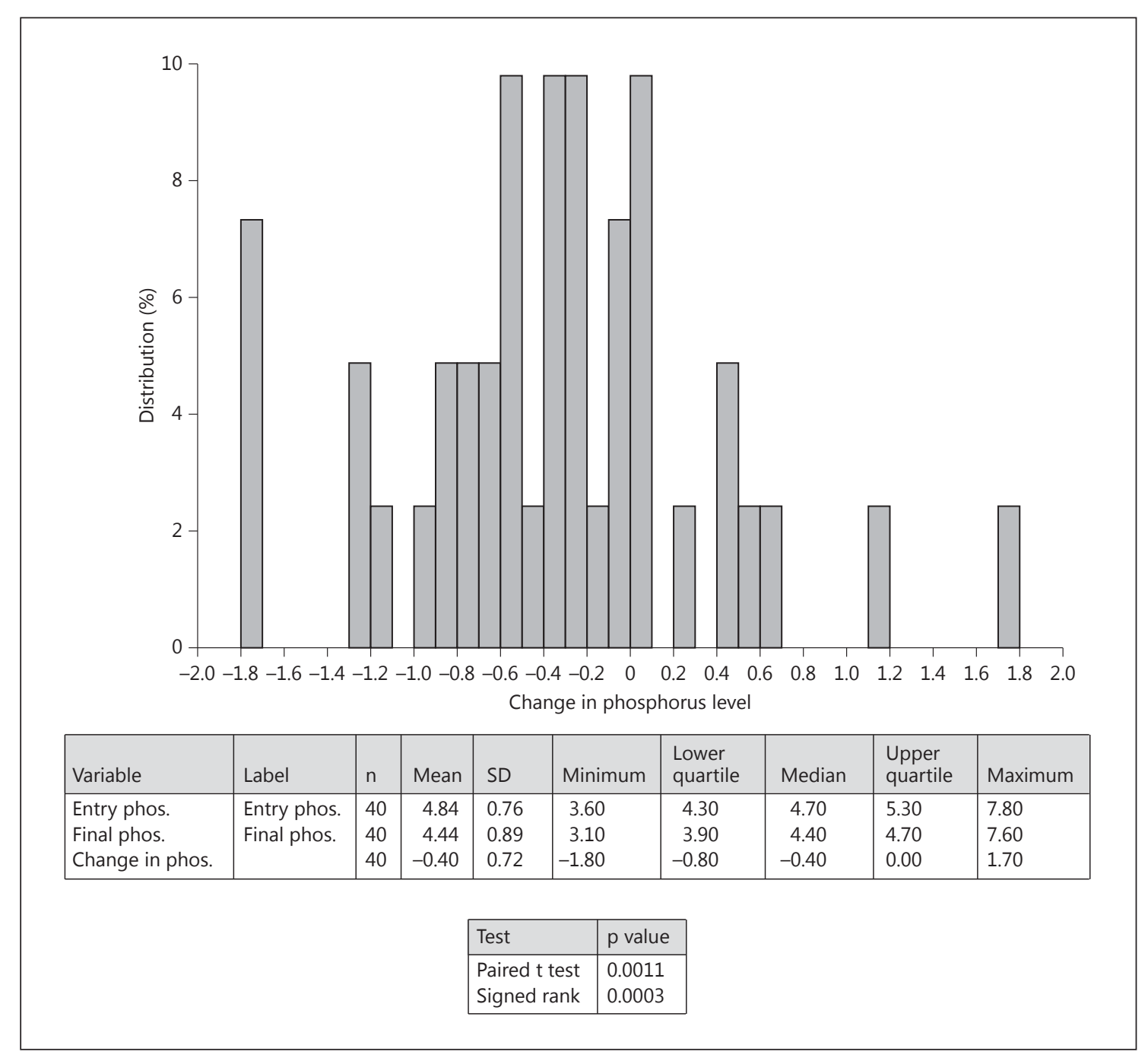

Fig. 1. Percent change in serum phosphorus levels after treatment with sevelamer carbonate for 3 months. Data are expressed as mean $\pm \mathrm{SD}$.

results. A recent study has shown that phosphorus reduction with sevelamer did have an effect on intact FGF23 but no effect on C-terminal FGF23, and thus our results may have differed depending on the use of the intact FGF23 assay [21].

One of the significant strengths of our study was the duration of treatment. This study was conducted over 3 months because it was suspected that hyperphosphatemic patients may require more prolonged therapy to achieve a reduction in FGF23. Even with sustained treatment, only one episode of hypophosphatemia developed which promptly resolved with discontinuation. An additional strength of our study was the limited exclusion criteria. Our study included relatively stable advanced CKD patients with only 3 patients requiring hospitalization during the study with very brief interruption of sevelamer.

This study had several limitations, including the design without randomization and control group. There was no formal assessment of dietary intake and compliance was not readily monitored for this study, which may explain the lack of effect on FGF23. Although this study did not collect 24-hour urine for phosphorus, we expect a decrease as the serum phosphorus decreased. One additional limitation was the use of the C-terminal FGF23 discussed above, which may have impacted our findings. 


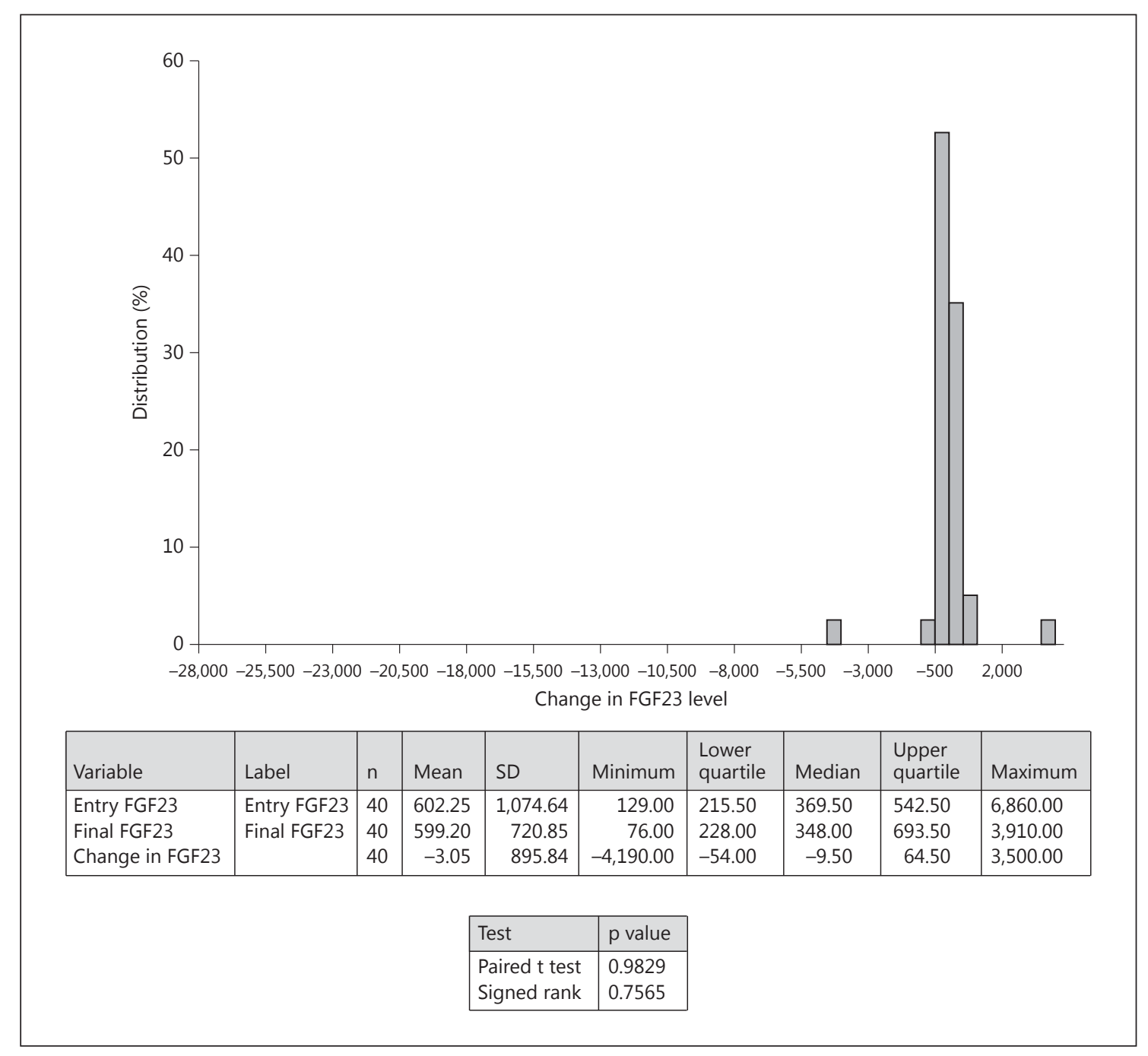

Fig. 2. Percent change in plasma FGF23 levels after treatment with sevelamer carbonate for 3 months. Data are expressed as mean $\pm \mathrm{SD}$.

In conclusion, the use of sevelamer carbonate in CKD patients with hyperphosphatemia was associated with reduction in serum phosphorus without a significant reduction in FGF23. Our results may suggest that using phosphate binders once hyperphosphatemia develops may be too late in the pathogenesis to have an impact. The conclusions from this study should ultimately be confirmed with a larger, prospective randomized study.

\section{Acknowledgement}

Genzyme Corporation provided the funding for this study.

\section{Disclosure Statement}

The authors have no conflicts of interest to disclose.

References $\quad 1$ Giachelli CM: The emerging role of phosphate in vascular calcification. Kidney Int 2009;75:890-897.

-2 Neves KR, et al: Adverse effects of hyperphosphatemia on myocardial hypertrophy, renal function, and bone in rats with renal failure. Kidney Int 2004;66:2237-2244.

3 Tonelli $\mathrm{M}$, et al: Relation between serum phosphate level and cardiovascular event rate in people with coronary disease. Circulation 2005;112:2627-2633. 
4 Kestenbaum B, et al: Serum phosphate levels and mortality risk among people with chronic kidney disease. J Am Soc Nephrol 2005; 16 : 520-528.

$>5$ Schiavi SC: Fibroblast growth factor 23: the making of a hormone. Kidney Int 2006;69: 425-427.

6 Fukumoto S, Yamashita T: FGF23 is a hormone-regulating phosphate metabolism unique biological characteristics of FGF23. Bone 2007;40:1190-1195.

7 Gutierrez O, et al: Fibroblast growth factor 23 mitigates hyperphosphatemia but accentuates calcitriol deficiency in chronic kidney disease. J Am Soc Nephrol 2005;16:220522015.

8 Isakova T, et al: Fibroblast growth factor 23 is elevated before parathyroid hormone and phosphate in chronic kidney disease. Kidney Int 2011;79:1370-1378.

-9 Isakova T, et al: Postprandial mineral metabolism and secondary hyperparathyroidism in early CKD. J Am Soc Nephrol 2008;19:615623.
10 El-Hajj Fuleihan G, et al: The parathyroid hormone circadian rhythm is truly endogenous - a general clinical research center study. J Clin Endocrinol Metab 1997;82:281-286.

11 Larsson T, et al: Circulating concentration of FGF23 increases as renal function declines in patients with chronic kidney disease, but does not change in response to variation in phosphate intake in healthy volunteers. Kidney Int 2003;64:2272-2279.

12 Gutierrez OM, et al: Fibroblast growth factor 23 and mortality among patients undergoing hemodialysis. N Engl J Med 2008;359:584592.

13 Wolf M, et al: Elevated fibroblast growth factor 23 is a risk factor for kidney transplant loss and mortality. J Am Soc Nephrol 2011;22: 956-966.

14 Isakova T, et al: Fibroblast growth factor 23 and risks of mortality and end-stage renal disease in patients with chronic kidney disease. JAMA 2011;305:2432-2439.

15 Fliser D, et al: Fibroblast growth factor 23 predicts progression of chronic kidney disease: the Mild to Moderate Kidney Disease (MMKD) Study. J Am Soc Nephrol 2007;18: 2600-2608.
6 Koiwa F, et al: Sevelamer hydrochloride and calcium bicarbonate reduce serum fibroblast growth factor 23 levels in dialysis patients. Ther Apher Dial 2005;9:336-339.

17 Oliveira RB, et al: Early control of PTH and FGF23 in normophosphatemic CKD patients: a new target in CKD-MBD therapy? Clin J Am Soc Nephrol 2010;5:286-291.

18 Vervloet MG, et al: Effects of dietary phosphate and calcium intake on fibroblast growth factor-23. Clin J Am Soc Nephrol 2011;6:383389.

19 Isakova T, et al: Effects of dietary phosphate restriction and phosphate binders on FGF23 levels in CKD. Clin J Am Soc Nephrol 2013 (E-pub ahead of print).

20 Isakova T, et al: Pilot study of dietary phosphorus restriction and phosphorus binders to target fibroblast growth factor 23 in patients with chronic kidney disease. Nephrol Dial Transplant 2011;26:584-591.

21 Block GA, et al: Effects of phosphate binders in moderate CKD. J Am Soc Nephrol 2012;23: 1407-1415. 